\title{
INCIDENCIA DE LOS ACTIVADORES DE JUICIOS DE METAMEMORIA Y SUGERENCIAS DE ESTRATEGIAS EN EL APRENDIZAJE AUTÓNOMO ${ }^{1}$
}

\author{
Luis Facundo Maldonado Granados, Ph. D. \\ Director del Proyecto
}

\begin{abstract}
Develop skills for autonomous learning is a challenge for educational systems in the current knowledge society. In fact, as information and knowledge production increases update learning becomes a necessary condition for person and enterprise achievement. Three questions are at the core of this paper: a- if metamemory judgment activators increase problem solution; $b$ - If suggestion of strategy facilitates learning of problem solving; and $c$ - if strong strategy learning generalizes for handling new problems. An experimental study was conducted using discovery problems on spatial reasoning. 9 computer games were programmed to support 4 experimental conditions: a) With metammemory judgment activators and strategy suggestion, b) With meta-memory judgment activa tors and no strategy suggestion, c) Without meta-memory judgment activators and with strategy suggestion, and $d$ - With no meta-memory judgment activators and no strategy suggestion. 150 high school students were randomly assigned to the experimental conditions. Statistical analysis uses factor analysis of variance and regression analysis. A simulation program allows reproducing the sequence of transition states followed by the problem solvers and identifying solution strategies. Metamemory judgements activators effects are interpreted in the frame of a motivational micro-system. Strategy suggestion operates after some basic experience on the task environment and mainly after the first success of solving the problem. Strategy generalization is evident among the initial stages of the learning curve of different problems.
\end{abstract}

\section{INTRODUCCIÓN}

Algunos trabajos previos realizados por este grupo motivaron el desarrollo de este proyecto que apunta a entender la dinámica de los procesos metacognitivos en contextos de solución de problemas. Estos trabajos estudiaron la formación de la capacidad para

\footnotetext{
${ }^{1}$ Como resultado del contrato 135 de 1997 celebrado entre la Universidad Pedagógica Nacional y el Instituto para la Investigación Educativa y el Desarrollo Pedagógico - IDEP - se llevó a cabo la investigación de la cual se ocupa el presente artículo.

El equipo de investigación estuvo conformado por Luis Facundo Maldonado Granados, Director del Proyecto, profesor del Departamento de Tecnología de la Universidad Pedagógica Nacional, Nerey

Ortega del Castillo, Magister en Educación y Desarrollo Social, Luis Bayardo Sanabria Rodríguez, Magister en Tecnologías de la Información y profesor del Instituto Pedagógico Nacional, David Macías Rodríguez Licenciado en Matemáticas Jaime lbañez Ibáñez Licenciado en Matemáticas, Martha Rosa Rubio Salas Licenciada en Educación Especial y Oscar Hernán Fonseca Ramírez Licenciado en Química y como asesora en arte Addy Pino Licenciada en Arte y Diseño.

El informe completo se publica en el libro: Metacognición y razonamiento espacial en Juegos de Computador. Universidad Pedagógica Nacional e Instituto para la Investigación y Desarrollo Pedagógico. Santa Fe de Bogotá, D. C. Editorial Cooperativa del Magisterio, 1999.
} 
que las personas ordenen su propio entorno en beneficio de objetivos de aprendizaje libremente elegidos; los procesos de decisión cuando se navega en ambientes hipertextuales; la influencia de estructuras cognitivas en la solución de problemas de razonamiento; regulación de metacognición a través del feedback, animación mental y solución de problemas. A través de estos trabajos se muestra que la formación de la capacidad de autodirección en el aprendizaje es posible dentro de algunas condiciones mínimas.

El estudio que aquí presentamos se inscribe en las tendencias de pensamiento que intentan integrar avances de la Inteligencia Artificial y la Psicología Cognitiva con el propósito de comprender y desarrollar habilidades metacognitivas que favorezcan la autonomía en los procesos de aprendizaje.

Frente a la necesidad de conocimiento y a la superproducción de datos que caracterizan ja era de la información se han destacado dos estrategias que tratan de potenciar a los individuos y a las organizaciones para incrementar la generación de conocimiento: la una está constituida por el uso de técnicas de inteligencia artificial para encontrar regularidades y hacer generalizaciones a partir de base de datos (Fayyad, Piatetsky, Smith, 1996) y la otra, al desarrollo de habilidades de aprendizaje autónomo por parte de los individuos.

Los estudios de prospectiva visualizan el futuro de la sociedad exitosa con base en el conocimiento y el aprendizaje autónomo, dado que se requiere desarrollar la capacidad creativa e innovadora para adecuarse a las circunstancias cambiantes del mundo contemporáneo y reducir el costo y aumentar la velocidad de la actualización de las empresas para mantener niveles de competitividad deseables.

\section{MARCO TEÓRICO}

Stenberg (1984) incluye la metacognición como una dimensión de la inteligencia. Se ha encontrado que si se comparan los estudiantes de bajo rendimiento con los de alto, estos últimos tienen repertorios más amplios de estrategias, son más flexibles en sus aproximaciones a la solución de los problemas, tienen más información sobre las circunstancias en las cuales las estrategias son apropiadas, valoran la relación entre esfuerzo y ejecución y monitorean con mayor cuidado el uso de las estrategias y la secuencia apropiada de actividades.

La metacognición es un tipo de conocimiento que toma como objeto un conocimiento adquirido. Los activadores de juicios de metamemoria son expresiones que solicitan a un aprendiz una valoración y un monitoreo sobre su conocimiento. Nelson \& Narens (1990) distinguen el monitoreo retrospectivo (el juicio sobre una respuesta previa) y el monitoreo prospectivo (juicio a cerca de futuras respuestas). El monitoreo prospectivo comprende: juicios a cerca de la facilidad de aprendizaje, emitidos previamente a la adquisición de un aprendizaje; juicios acerca del aprendizaje y que se emiten durante o después de un aprendizaje acerca de la ejecución futura de ese aprendizaje y juicios acerca de ítems que no se recuerdan en el momento y que valoran si se tienen o no aprendizajes que ya se aprendieron o se están aprendiendo.

Maldonado y Andrade (1996) encuentran que en ambientes de juegos de descubrimiento basados en computador los estudiantes que tienen activadores de juicios de metamemoria ponen en ejecución estrategias de solución de problemas con una probabilidad mayor que aquellos que no disponen de estos activadores y que hay 
correlación positiva entre desarrollo de estrategias y retención de conceptos, pero que ésta disminuye cuando se presentan problemas nuevos y sugieren la hipótesis de que los estudiantes que usan estrategias fuertes podrán desarrollar mayor retención de conceptos. En estas condiciones surge la necesidad de un modelo coherente que permita interpretar la dinámica de la transferencia de estrategias.

\section{RESUMEN DE ESPECIFICACIONES METODOLÓGICAS}

La investigación se guié por tres preguntas:

1. ¿Existe diferencia significativa en La cantidad de problemas resueltos por unidad de tiempo entre un grupo de estudiantes que usan un ambiente activador de juicios metacognitivos sobre memoria y otro que usa un ambiente no activador de estos juicios?

2. ¿Existen diferencias en cuanto a cantidad de estrategias usadas por un grupo que es entrenado en un ambiente que exige escogencia de estrategias con respecto a otro grupo que no tiene esa experiencia de aprendizaje?

3. ¿Existen diferencias significativas en pruebas de generalización entre estudiantes que desarrollan estrategias fuertes de solución de problemas y aquellos que no desarrollan estas estrategias?

Se escogieron como variables dependientes la Eficacia y la Eficiencia, las cuales se integran en el constructo Rendimiento. La primera se define como la relación entre eventos exitosos y el total de eventos invertidos en la solución de un problema y la segunda como la relación entre eventos exitosos y la cantidad de tiempo requerido para resolverlo. Para responder a la pregunta cuánto rinde por unidad de tiempo se introdujo el concepto de Rendimiento definido como la eficacia dividida por la cantidad de tiempo. Al integrar eficacia y tiempo se tiene un concepto que integra la Eficacia con La Eficiencia.

Se Desarrollaron 9 juegos basados en computador sobre temas de razonamiento espacial y diseño. El computador se programó para que hiciera Los registros de datos y genera un protocolo que simulara os procesos seguidos por los sujetos en la solución de los problemas. El programa se desarrolló en el lenguaje Open Script de Toolbook CBT versión $4.0^{2}$.

150 estudiantes de décimo y undécimo grado de educación vocacional del Instituto Pedagógico Nacional anexo a la Universidad Pedagógica Nacional de Bogotá fueron asignados de manera aleatoria a cuatro condiciones experimentales y desarrollaron las sesiones de aprendizaje en dos jornadas de 6 horas con un intermedio de dos días entre sesión.

Los datos se analizaron tanto en forma cuantitativa como cualitativa. En el análisis cuantitativo se aplicaron dos métodos estadísticos: un análisis factorial de varianza para evaluar el efecto de los activadores de juicios de metamemoria y La sugerencia de estrategias sobre las tres variables dependientes por separado y un análisis de regresión múltiple para evacuar La generalización de estrategias entre parejas de juegos. El análisis cualitativo se hace con base en la ejecución de los simuladores para un número más limitado de sujetos en dos de los nueve juegos y realiza una descripción a profundidad de los mismos aspectos estudiados de manera global parlas modelos estadísticos. 


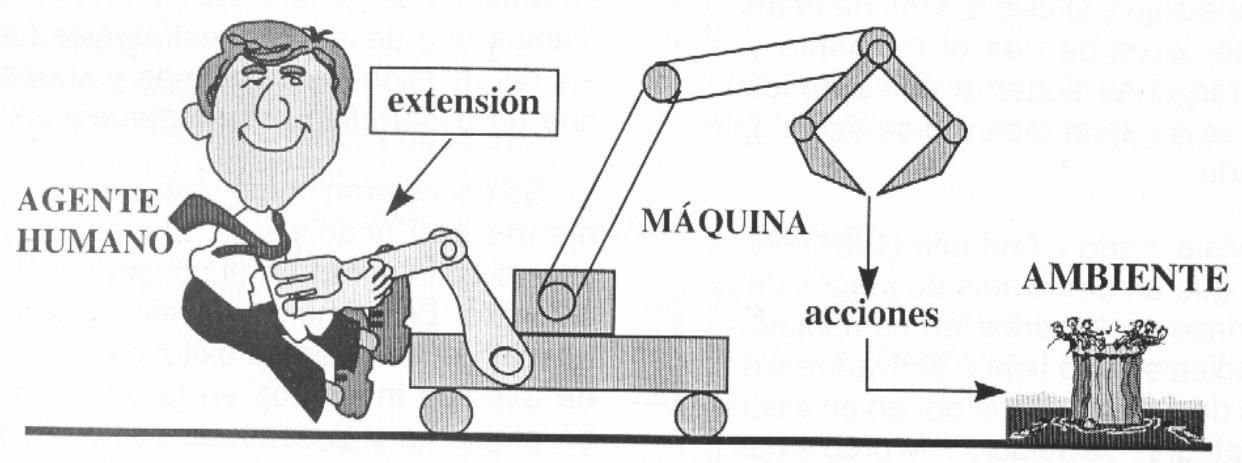

Ilustración 1: Estructura de Grúa en el juego sobre mecanismos.

\section{ANÁLISIS CUALITATIVO DE RESULTADOS}

El análisis cualitativo basado en el uso de simuladores se aplicó a dos juegos uno sobre ensamblaje de mecanismos y otro sobre orientación:

El juego consistía en armar una grúa para trasladar un objeto que el jugador elegía de tres disponible y que diferían en forma y disposición. El ensamblaje de la máquina se hacía con piezas que podía adquirir en un almacén. Como activador de juicio de metamemoria se utilizó el proceso de compra de piezas con un presupuesto fijo. Las piezas tenían un valor y la consulta de información - posible a través de un mecanismo de ayuda en línea- también tenía costo. La sugerencia de estrategias para el grupo que podía usarlas se hacía presentándolas al iniciar el juego, obligando al jugador a leerlas y, además, podía consultarlas cada vez que así lo deseaba.

Los sujetos del grupo que no contaron con estrategias ni con juicios de metamemoria en primera instancia exploraron al azar las diferentes piezas dispuestas en el almacén. No revisaron la ayuda como punto de orientación para el proceso de búsqueda de solución. El modelo de máquina que lograron conceptualizar no fue muy diferente al que los sujetos tenían antes de enfrentar el problema. Su representación después de resolver los tres primeros juegos los llevó a ver la máquina solamente como una estructura con brazos.

En el grupo B, que a diferencia del anterior contó con activadores de juicios de metamemoria, los datos posibilitan ver con claridad que en una primera instancia los juicios no fueron tenidos en cuenta en las decisiones de los eventos que pretendían desarrollar los sujetos. En los dos juegos siguientes ya se observa la emisión de juicios y se muestra una mejor planeación y dosificación de eventos. Conceptualmente, a diferencia del anterior, afinan la concepción de máquina a partir de La consulta de información técnica de las piezas y forman la idea de máquina como una estructura de agarre. 
Tabla 1. Estrategias desarrolladas parles sujetos del grupo $A$.

\begin{tabular}{|c|c|c|c|}
\hline $\begin{array}{l}\text { ESTRATEGIA } \\
\text { SEGUIDA POR } \\
\text { EL SUJETO }\end{array}$ & $\begin{array}{l}\text { CARACTERIZACIÓN } \\
\text { DE LA ESTRATEGIA }\end{array}$ & $\begin{array}{l}\text { EVENTOS FUERTES } \\
\text { DE LA ESTRATEGIA }\end{array}$ & $\begin{array}{l}\text { CONCEPCIÓN } \\
\text { DE MÁQUINA } \\
\text { POR EL SUJETO }\end{array}$ \\
\hline $\begin{array}{l}\text { comparación de } \\
\text { piezas almacén, }\end{array}$ & $\begin{array}{l}\text { Comparación de la } \\
\text { forma de una pieza con } \\
\text { respecto a otra. }\end{array}$ & $\begin{array}{l}\text { Clic en la pieza patrón, } \\
\text { clic en la pieza a com- } \\
\text { parar. }\end{array}$ & \multirow{4}{*}{$\begin{array}{l}\text { ESTRUCTURA } \\
\text { CONFORMADA } \\
\text { POR BRAZOS Y } \\
\text { MECANISMOS } \\
\text { DE ENGANCHE }\end{array}$} \\
\hline $\begin{array}{l}\text { Acumulación de } \\
\text { información, } \\
\text { Para comprar } \\
\text { pieza. }\end{array}$ & $\begin{array}{l}\text { Consulta ayuda de la } \\
\text { pieza seleccionada. }\end{array}$ & \begin{tabular}{lrr} 
Clic para & \multicolumn{2}{c}{ seleccionar } \\
pieza; clic & para \\
acceder a la & lnformación técnica
\end{tabular} & \\
\hline $\begin{array}{l}\text { Verificación de } \\
\text { las piezas } \\
\text { componentes de } \\
\text { la máquina, }\end{array}$ & $\begin{array}{l}\text { Comprar el mayor } \\
\text { número de piezas y } \\
\text { verificar si son o no de } \\
\text { la máquina. }\end{array}$ & $\begin{array}{l}\text { Clic en la pieza y clic } \\
\text { en el botón devolución. }\end{array}$ & \\
\hline $\begin{array}{ll}\text { Ensamblaje } & \text { de } \\
\text { piezas en los } \\
\text { puntos } & \\
\text { estructurales. } & \end{array}$ & $\begin{array}{l}\text { Comprar, desplazar y } \\
\text { probar la pieza en cada } \\
\text { uno de los puntos } \\
\text { estructurales. }\end{array}$ & $\begin{array}{l}\text { Clic en la pieza a com- } \\
\text { prar, clic sostenido } \\
\text { para desplazar la pieza } \\
\text { y soltar la pieza en el } \\
\text { punto de articulación } \\
\text { para probarla. }\end{array}$ & \\
\hline $\begin{array}{lr}\text { Identificación } & \text { del } \\
\text { objeto } & \text { a } \\
\text { desplazar } & \text { y } \\
\text { estructuración } & \\
\text { mental } & \text { del } \\
\text { modelo } & \text { de } \\
\text { máquina. } & \end{array}$ & $\begin{array}{l}\text { Compara los objetos a } \\
\text { desplazar y toma la } \\
\text { decisión del mecanismo } \\
\text { a construir. }\end{array}$ & $\begin{array}{l}\text { Desplaza el cursor so- } \\
\text { bre los objetos. Clic en } \\
\text { las reglas del juego. } \\
\text { Clic en el objeto } \\
\text { seleccionado. } \\
\text { Clic en piezas que co- } \\
\text { rresponden al meca- } \\
\text { nismo. } \\
\text { Clic sostenido para } \\
\text { desplazar y probar las } \\
\text { piezas en los puntos } \\
\text { estructurales. }\end{array}$ & $\begin{array}{l}\text { ESTRUCTURA } \\
\text { CONFORMADA } \\
\text { POR } \\
\text { ELEMENTOS } \\
\text { PREVIOS } \\
\text { MANEJADOS } \\
\text { POR } \\
\text { SUJETOS: LOS } \\
\text { BRAZOS } \\
\text { POLEAS }\end{array}$ \\
\hline $\begin{array}{lr}\text { Ensamblaje } & \text { de } \\
\text { dos tipos } & \text { de } \\
\text { piezas: las } & \text { de } \\
\text { soporte } & \text { y } \\
\text { rnovimiento. } & \end{array}$ & $\begin{array}{l}\text { Ubica en forma } \\
\text { intercalada una pieza de } \\
\text { soporte y una de } \\
\text { movimiento. }\end{array}$ & $\begin{array}{l}\text { Clic para seleccionar } \\
\text { pieza de soporte. Clic } \\
\text { sostenido para ubicarla } \\
\text { en el punto estructural. } \\
\text { Clic para seleccionar } \\
\text { pieza de movimiento y } \\
\text { colocarlo en el punto } \\
\text { definido. }\end{array}$ & $\begin{array}{l}\text { ESTRUCTURA } \\
\text { CONFORMADA } \\
\text { POR PIEZAS DE } \\
\text { SOPORTE r Y } \\
\text { PIEZAS YUE } \\
\text { GENERAN } \\
\text { MOVIMIENTO }\end{array}$ \\
\hline
\end{tabular}


Estos dos grupos en la etapa de generalización caracterizada en las Tablas 1y 2 nos permiten inferir que:

- Las estrategias que construían los sujetos de los grupos A y B estaban centradas en la forma de las piezas y en la compra de todas ellas. Con respecto al tiempo, los datos reflejan preocupación por La eficiencia, es decir, importaba gastar menos tiempo en los eventos sin contar con la planeación del éxito del procedimiento.

- Las nuevas producciones aparecen a partir del segundo juego a través de la clasificación entre piezas de movimiento y de soporte. Las producciones viejas se reflejaron en la repetición de los eventos para comprar la totalidad de las piezas sin criterio.

Tabla 2. Estrategias desarro0adas por/os sujetos de/grupo E.

\begin{tabular}{|c|c|c|c|c|}
\hline $\begin{array}{l}\text { ESTRATEGIA } \\
\text { SEGUIDA } \\
\text { POR } \\
\text { SUJETO }\end{array}$ & $\begin{array}{l}\text { CARACTERIZA- } \\
\text { CIÓN DE LA } \\
\text { ESTRATEGIA }\end{array}$ & $\begin{array}{l}\text { JUICIO DE } \\
\text { METAMEMORIA }\end{array}$ & $\begin{array}{l}\text { EVENTOS } \\
\text { FUERTES DE } \\
\text { LA } \\
\text { ESTRATEGIA }\end{array}$ & $\begin{array}{l}\text { CONCEPCIÓN } \\
\text { DE MÁQUINA } \\
\text { POR EL } \\
\text { SUJETO }\end{array}$ \\
\hline $\begin{array}{l}\text { identificación } \\
\text { del objeto a } \\
\text { desplazar y } \\
\text { estructuración } \\
\text { mental del } \\
\text { modelo de } \\
\text { máquina }\end{array}$ & $\begin{array}{lr}\text { Compara } & \text { los } \\
\text { objetos a } & \text { a } \\
\text { desplazar y toma } \\
\text { la decisión de las } \\
\text { piezas ra } \\
\text { considera son la } \\
\text { máquina, }\end{array}$ & $\begin{array}{l}\text { No afecté } \\
\text { ninguna de las } \\
\text { decisiones } \\
\text { tomadas por los } \\
\text { asistes en de la } \\
\text { construcción de la } \\
\text { solución del } \\
\text { problema. }\end{array}$ & $\begin{array}{l}\text { Desplaza el } \\
\text { cursor acore los } \\
\text { objetos. Clic en } \\
\text { las reglas del } \\
\text { luego. Clic en el } \\
\text { objeto } \\
\text { seleccionado. } \\
\text { Clic en piezas } \\
\text { que } \\
\text { corresponden al } \\
\text { mecanismo } \\
\text { Clic sostenido } \\
\text { para desplazar } \\
\text { y probar las } \\
\text { piezas en los } \\
\text { puntos } \\
\text { estructurales. }\end{array}$ & $\begin{array}{l}\text { NO } \\
\text { CONSOLIDA } \\
\text { UNA } \\
\text { ESTRUCTURA } \\
\text { CLARA DE LA } \\
\text { MÁOUINA QUE } \\
\text { DEBE } \\
\text { CONSTRUIR }\end{array}$ \\
\hline $\begin{array}{l}\text { Relacione la } \\
\text { forma cbjeto a } \\
\text { movilizar y la } \\
\text { pieza de la } \\
\text { máquina } \\
\text { necesaria para } \\
\text { sujetarlo }\end{array}$ & $\begin{array}{l}\text { Prueba en los } \\
\text { puntos } \\
\text { estructurales la } \\
\text { pieza } \\
\text { seleccionada } \\
\text { para sujetar el } \\
\text { objeto. Intercala } \\
\text { otras piezas y } \\
\text { sigue probando la } \\
\text { funcionalidad con } \\
\text { la primera pieza. }\end{array}$ & $\begin{array}{l}\text { El juicio influyó } \\
\text { notoriamente en } \\
\text { las decisiones de } \\
\text { los sujetes con } \\
\text { respecto a: } \\
\text { manejo de } \\
\text { información. } \\
\text { (Invirtió en ayuda } \\
20.000 \text { ) compra } \\
\text { de piezas (gastó } \\
345.000\end{array}$ & $\begin{array}{l}\text { Clic en la pieza } \\
\text { de sujeción. } \\
\text { Clic en una } \\
\text { segunda pieza. } \\
\text { clic sostenido } \\
\text { para armar las } \\
\text { dos piezas en } \\
\text { los puntos } \\
\text { estructurales. } \\
\text { Clic sostenido } \\
\text { para intercalar } \\
\text { una nueva }\end{array}$ & $\begin{array}{l}\text { AFINA SU } \\
\text { PROPIA } \\
\text { CONCEPCIÓN } \\
\text { DE MÁOUINA A } \\
\text { PARTIR DE LA } \\
\text { ULTIMA PIEZA } \\
\text { CONSIDERADA } \\
\text { ESTADO FINAL } \\
\text { DEL } \\
\text { PROBLEMA }\end{array}$ \\
\hline
\end{tabular}




\begin{tabular}{|c|c|c|c|c|}
\hline & & $\begin{array}{l}\text { devolución de } \\
\text { éstas (30.000). } \\
\text { Finalmente } \\
\text { obtuvo ganancia } \\
\text { por valor de } \\
605.000 .\end{array}$ & $\begin{array}{l}\text { pieza. Clic } \\
\text { sostenido para } \\
\text { probar la pieza } \\
\text { de sujeción } \\
\text { seleccionada. }\end{array}$ & \\
\hline $\begin{array}{l}\text { Identifica los } \\
\text { puntos } \\
\text { estructurales } \\
\text { del bastidor y } \\
\text { mentalmente } \\
\text { las relaciona } \\
\text { con las piezas } \\
\text { que considera } \\
\text { son del } \\
\text { mecanismo. }\end{array}$ & $\begin{array}{lr}\text { Desarrolla } & \text { un } \\
\text { plan de ensamble } \\
\text { a partir de la } \\
\text { identificación del } \\
\text { bastidor y el } \\
\text { señalamiento sin } \\
\text { selección de las } \\
\text { piezas que } \\
\text { considera debe } \\
\text { adquirir. Consulta } \\
\text { información sin } \\
\text { costo antes de la } \\
\text { compra de cada } \\
\text { pieza. }\end{array}$ & $\begin{array}{l}\text { Para estos } \\
\text { sujetos el juicio } \\
\text { exigió la } \\
\text { priorización del } \\
\text { factor de compra } \\
\text { de piezas, donde } \\
\text { invirtió el dinero } \\
\text { gastado. }\end{array}$ & \begin{tabular}{lr}
\multicolumn{3}{c}{ Clic en el objeto } \\
a movilizar. \\
Señalar con el \\
cursor las \\
piezas que \\
compraría. & Clic \\
para ir al sitio \\
de ensamble. \\
ldentifica con el \\
cursor \\
puntos los \\
estructurales. \\
Clic en la pieza \\
a comprar. Clic \\
en ayuda para \\
obtener \\
información de \\
la pieza se- \\
leccionada Clic \\
para adquirir la \\
pieza. \\
sostenido plic \\
ubicar la pieza.
\end{tabular} & $\begin{array}{l}\text { CONSTRUYE } \\
\text { UNA } \\
\text { CONCEPCIÓN } \\
\text { DE MAOUINA A } \\
\text { PARTIR DE } \\
\text { INFORMACIÓN } \\
\text { DE CADA UNA } \\
\text { DE LAS PIEZAS }\end{array}$ \\
\hline
\end{tabular}

Las tablas 3 y 4 muestran los datos correspondientes a los grupos $C$ y $D$, a partir de los cuales hacemos las siguientes inferencias:

Tabla 3a. Estrategias desarrolladas por los sujetos del grupo C.

\begin{tabular}{|c|c|c|c|c|}
\hline $\begin{array}{l}\text { SELECCIÓN } \\
\text { DE LAS } \\
\text { ESTRATEGIAS }\end{array}$ & $\begin{array}{l}\text { CARACTERIZA- } \\
\text { CIÓN DE LA } \\
\text { ESTRATEGIA }\end{array}$ & $\begin{array}{l}\text { ESTRATEGIA } \\
\text { SEGUIDA POR } \\
\text { EL SUJETO }\end{array}$ & $\begin{array}{l}\text { EVENTOS } \\
\text { FUERTES DE } \\
\text { LA } \\
\text { ESTRATEGIA* }\end{array}$ & $\begin{array}{l}\text { CONCEPCIÓN } \\
\text { DE MÁQUINA } \\
\text { DEL SUJETO }\end{array}$ \\
\hline \multicolumn{5}{|c|}{$\begin{array}{l}6 \text { SUJETOS } \\
\text { Desarrollaron la siguiente secuencia de utilización de estrategias. }\end{array}$} \\
\hline $\begin{array}{lr}\text { Para el primer } \\
\text { juego } \\
\text { seleccionaron la } \\
\text { estrategia } 1 \\
\text { basados en la } \\
\text { forma de la } \\
\text { máquina, }\end{array}$ & $\begin{array}{l}\text { Identi6cación de } \\
\text { la in- formación } \\
\text { sin costo, } \\
\text { Adquisición dalas } \\
\text { piezas de } \\
\text { acuerdo con la } \\
\text { forma del objeto }\end{array}$ & 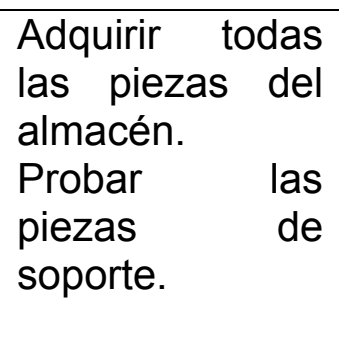 & $\begin{array}{l}\text { Tomar una } \\
\text { pieza con punto } \\
\text { de referencia. } \\
\text { Tomar el botón } \\
\text { devolución. }\end{array}$ & \\
\hline
\end{tabular}

* Determina el camino de solución. Aquí el sujeto entiende el problema y su solución. 
UNIVERSIDAD PEDAGÓGICA NACIONAL

\begin{tabular}{|c|c|c|c|c|}
\hline $\begin{array}{l}\text { En el segundo } \\
\text { juego toma la } \\
\text { estrategia tres, }\end{array}$ & $\begin{array}{l}\text { Estructuración } \\
\text { plan de } \\
\text { ensamble. } \\
\text { Utilización de la } \\
\text { opción } \\
\text { devolución. }\end{array}$ & $\begin{array}{lr}\text { Ubicar } & \text { una } \\
\text { pieza } & \text { para } \\
\text { tomarla } & \text { como } \\
\text { punto } & \text { de } \\
\text { referencia } & \text { de } \\
\text { otras } & \\
\text { semejantes } & \end{array}$ & $\begin{array}{l}\text { Selecciona una } \\
\text { pieza y verifica } \\
\text { la información } \\
\text { con el botón de } \\
\text { información } \\
\text { técnica, }\end{array}$ & $\begin{array}{lr}\text { Trata } & \text { de } \\
\text { consolidar una } \\
\text { concepción de } \\
\text { máquina como } \\
\text { una }\end{array}$ \\
\hline $\begin{array}{l}\text { En el tercer } \\
\text { juego intentan } \\
\text { nuevamente } \\
\text { con la primera } \\
\text { estrategia. }\end{array}$ & $\begin{array}{l}\text { Identificación de } \\
\text { las piezas a } \\
\text { través del manejo } \\
\text { de la ayuda y } \\
\text { utilización de la } \\
\text { opción } \\
\text { devolución. }\end{array}$ & $\begin{array}{ll}\text { Acentúa } & \text { el } \\
\text { manejo de la } \\
\text { información } \\
\text { para adquirir } \\
\text { las piezas. }\end{array}$ & & $\begin{array}{l}\text { Identifica } \\
\text { movimiento y } \\
\text { soporte de las } \\
\text { piezas. }\end{array}$ \\
\hline
\end{tabular}

Tabla 3b. Estrategias desarrolladas por/os sujetos del grupo $C$.

\begin{tabular}{|l|l|l|l|l|}
\hline SELECCIÓN & CARACTERIZA- \\
DE LAS & CIÓN DE LA & ESTRATEGIA & EVENTOS & CONCEPCIÓN \\
ESTRATEGIAS & ESTRATEGIA & $\begin{array}{l}\text { ESUEA POR } \\
\text { EL SUJETO }\end{array}$ & $\begin{array}{l}\text { FUERTES DE } \\
\text { LA }\end{array}$ & $\begin{array}{l}\text { DE MÁQUINA } \\
\text { ESTRATEGIA* SUJETO }\end{array}$ \\
\hline
\end{tabular}

7 SUJETOS avanzan en la solución del problema de acuerdo con la utilización de la siguiente orden de estrategias

\begin{tabular}{|c|c|c|c|c|}
\hline $\begin{array}{lr}\text { Primer juego, } \\
\text { estrategia } \\
\text { seleccionada la } \\
\text { número } & 1 \\
\text { basada en el } \\
\text { manejo } & \text { de } \\
\text { información. }\end{array}$ & \begin{tabular}{l} 
Conocimiento de \\
la información a \\
partir del botón \\
ayuda. \\
\multicolumn{4}{l}{ Organización de } \\
las piezas \\
seleccionadas a \\
parir del objeto a \\
mover
\end{tabular} & $\begin{array}{l}\text { Selección de la } \\
\text { pieza y } \\
\text { conocimiento de } \\
\text { la Información } \\
\text { técnica, } \\
\text { Realiza plan de } \\
\text { ensamble a } \\
\text { partir del botón } \\
\text { evolución. } \\
\text { Ensambla } \\
\text { piezas de } \\
\text { soporte y las } \\
\text { combina con las } \\
\text { de movimiento. }\end{array}$ & $\begin{array}{l}\text { Selección de } \\
\text { las piezas } \\
\text { propias de la } \\
\text { máquina a partir } \\
\text { del botón } \\
\text { evolución }\end{array}$ & $\begin{array}{l}\text { Maneja el } \\
\text { concepto } \\
\text { máquina como } \\
\text { un sistema } \\
\text { compuesto por } \\
\text { elementos de } \\
\text { soporte y } \\
\text { dinámicos. }\end{array}$ \\
\hline $\begin{array}{l}\text { Segundo juego, } \\
\text { estrategia } \\
\text { utilizada la } \\
\text { número } 2 . \\
\text { basada en la } \\
\text { forma de las } \\
\text { piezas finales } \\
\text { de la máquina y } \\
\text { la del objeto a } \\
\text { movilizar. }\end{array}$ & $\begin{array}{l}\text { Identificación y } \\
\text { reconocimiento } \\
\text { de la forma del } \\
\text { objeto. Selección } \\
\text { de piezas que } \\
\text { permitan r el } \\
\text { agarre del objeto } \\
\text { ldentificación de } \\
\text { la utilidad del } \\
\text { botón devolución. }\end{array}$ & $\begin{array}{l}\text { Busca } \\
\text { ensamblar las } \\
\text { piezas por fuera } \\
\text { de los puntos } \\
\text { estructurales, } \\
\text { Pruebar las } \\
\text { piezas cuya } \\
\text { función es la de } \\
\text { agarrer del } \\
\text { objeto. Ubica el }\end{array}$ & $\begin{array}{l}\text { Identificación } \\
\text { del botón ayuda } \\
\text { y el manejo de } \\
\text { la información } \\
\text { antes de } \\
\text { adquirir la pieza } \\
\text { seleccionada. }\end{array}$ & $\begin{array}{l}\text { Proyecta dos } \\
\text { concepciones } \\
\text { de máquina: la } \\
\text { primera basada } \\
\text { en la integración } \\
\text { del objeto la } \\
\text { cuerda y las pie- } \\
\text { zas da agarre; } \\
\text { en la segunda } \\
\text { toma los }\end{array}$ \\
\hline
\end{tabular}




\begin{tabular}{|c|c|c|c|c|}
\hline & & $\begin{array}{lr}\text { espacio } & \text { de } \\
\text { construcción } & \text { da } \\
\text { la máquina a } \\
\text { partir del } & \text { sitio } \\
\text { donde } & \text { está } \\
\text { ubicado } & \text { el } \\
\text { objeto. } & \end{array}$ & & $\begin{array}{l}\text { elementos } \\
\text { anteriores pero } \\
\text { los ubica en el } \\
\text { bastidor como } \\
\text { punto } \\
\text { estructural para } \\
\text { lograr } \\
\text { movimiento. }\end{array}$ \\
\hline $\begin{array}{l}\text { Para el tercer } \\
\text { intento } \\
\text { selecciona la } \\
\text { estrategia } 3 \text {, } \\
\text { fundamentada } \\
\text { en la prueba } \\
\text { dala pieza en } \\
\text { los puntos } \\
\text { estructurales del } \\
\text { bastidor. }\end{array}$ & $\begin{array}{l}\text { Identificación de } \\
\text { las piezas a } \\
\text { través del manejo } \\
\text { de la ayuda y } \\
\text { utilización de la } \\
\text { opción } \\
\text { devolución. }\end{array}$ & 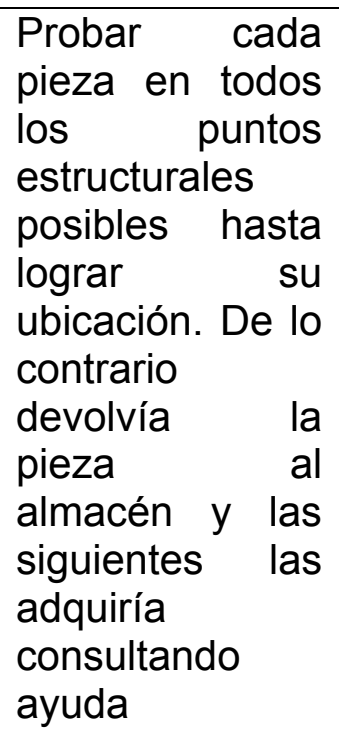 & $\begin{array}{lr}\text { Probar } & \text { cada } \\
\text { pieza en los } \\
\text { puntos } \\
\text { estructurales }\end{array}$ & $\begin{array}{lr}\text { Trato } & \text { de } \\
\text { consolidar } & \text { el } \\
\text { concepto } & \text { de } \\
\text { sistema } & \text { de } \\
\text { movimiento } & \text { y } \\
\text { enganche. } & \end{array}$ \\
\hline $\begin{array}{l}8 \text { SUJETOS } \\
\text { determinaron } \\
\text { trabajar los tres } \\
\text { juegos con la } \\
\text { misma } \\
\text { estrategia, se } \\
\text { analiza con la } \\
\text { primera. }\end{array}$ & $\begin{array}{lrr}\text { Forma } & & y \\
\text { estructura de la } \\
\text { máquina. } & & \\
& \end{array}$ & $\begin{array}{l}\text { Recorrieron } \\
\text { todas las veces } \\
\text { los puntos } \\
\text { estructurales del } \\
\text { bastidor y de } \\
\text { ellas mismas } \\
\text { tratando de } \\
\text { ensamblarlas de } \\
\text { acuerdo con la } \\
\text { forma. }\end{array}$ & $\begin{array}{lr}\text { Clasificar las } \\
\text { Piezas de la } \\
\text { máquina } \\
\text { través a } \\
\text { Detén } \\
\text { devolución, }\end{array}$ & \begin{tabular}{lr}
\multicolumn{2}{c}{ El modelo de } \\
máquina lo \\
consolidan \\
partir de \\
concepción \\
enganche \\
objeto.
\end{tabular} \\
\hline
\end{tabular}

- En los primeros juegos es notoria la dificultad para seguir los procedimientos exigidos por la estrategia seleccionada. Estos se combinan con otros eventos que en ocasiones pertenecen a estrategias no seleccionadas. En cuanto a la concepción de máquina en el primer juego no se presentan diferencias con respecto a las de los grupos anteriores.

- En el segundo y el tercer juego los sujetos aprenden a seguir secuencias de eventos y logran desarrollar en forma más estricta la estrategia seleccionada. Con respecto a la construcción del concepto de máquina éste se amplía: entienden que existen varios tipos de máquina que cumplen funciones diferentes. Este resultado lo alcanzan después de identificar las formas de los objetos que se deben transportar.

- En la etapa de generalización estos sujetos logran identificar que es más exitoso trabajar con la misma estrategia con la cual realizaron los primeros juegos e identifican los eventos fuertes que transfieren. El concepto de máquina se construye identificando elementos de soporte y dinámicos que se enlazan para generar un primer mecanismo que se repite - a manera de subestructura - a medida que se avanza en la solución. 
- Los sujetos del grupo D demostraron en el primer juego una gran dificultad para articular los juicios de metamemoria con las estrategias. En el segundo y tercer juego logran una primera aproximación de relación entre estos dos elementos sobre la base de a retención de información, llegando a establecer una regularidad entre la estrategia seleccionada y el nivel de conocimiento reflejado en el manejo del juicio. La consolidación del modelo de máquina lo estructuran desde el inicio del juego: establecen una relación sistemática entre adquisición de información y compra de las piezas.

Tabla 4. Estrategias desarrolladas por los sujetos del grupo D.

\begin{tabular}{|c|c|c|c|}
\hline $\begin{array}{l}\text { ESTRATEGIA } \\
\text { SEGUIDA POR } \\
\text { EL SUJETO }\end{array}$ & $\begin{array}{l}\text { CARACTERIZACIÓN DE } \\
\text { LA ESTRATEGIA }\end{array}$ & $\begin{array}{l}\text { EVENTOS } \\
\text { FUERTES DE LA } \\
\text { ESTRATEGIA }\end{array}$ & $\begin{array}{l}\text { CONCEPCIÓN DE } \\
\text { MÁQUINA POR EL } \\
\text { SUJETO }\end{array}$ \\
\hline \multicolumn{4}{|l|}{ PRIMER JUEGO } \\
\hline $\begin{array}{l}\text { El juicio de } \\
\text { metamemoria en } \\
\text { el primer juego } \\
\text { refleja un alto } \\
\text { nivel de } \\
\text { conocimiento del } \\
\text { problema. } \\
\text { La estrategia } \\
\text { seleccionada es } \\
\text { la 2, } \\
\text { fundamentada } \\
\text { en el manejo de } \\
\text { la forma de las } \\
\text { piezas y el } \\
\text { objeto y } \\
\text { movilizar, a }\end{array}$ & 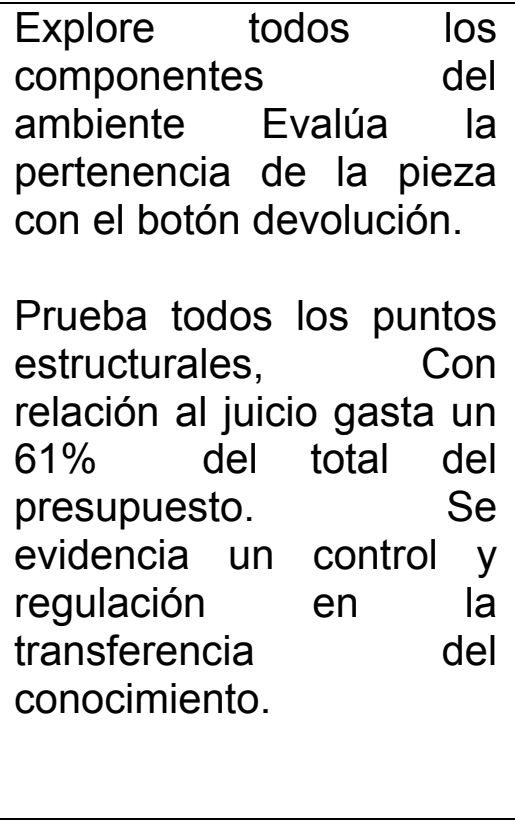 & $\begin{array}{l}\text { No se evidencia } \\
\text { una reacción sino } \\
\text { un manejo in } \\
\text { dependiente de } \\
\text { cada uno de estos } \\
\text { factores, }\end{array}$ & $\begin{array}{l}\text { Generaliza el éxito lo- } \\
\text { grado con la posición } \\
\text { de una pieza y lo pre- } \\
\text { tende transferir a } \\
\text { todas las piezas de la } \\
\text { misma forma. Avanza } \\
\text { en el conocimiento y } \\
\text { manejo a partir de la } \\
\text { evaluación de la } \\
\text { pertenencia o no de } \\
\text { las piezas. Indica un } \\
\text { alto nivel de } \\
\text { predicción. }\end{array}$ \\
\hline \multicolumn{4}{|c|}{ SEGUNDO JUEGO } \\
\hline $\begin{array}{ll}\text { Toma la } \\
\text { segunda con el } \\
\text { mismo criterio de } \\
\text { selección, }\end{array}$ & $\begin{array}{l}\text { Proyecta una retención } \\
\text { del conocimiento de los } \\
\text { eventos exitosos en un } \\
80 \% \text { del total de las } \\
\text { piezas a ubicar. } \\
\text { Controla el gasto y } \\
\text { reduce la inversión en } \\
70 \% \text { a partir de } \\
\text { seleccionar con mayor } \\
\text { precisión las piezas } \\
\text { propias de la máquina. } \\
\text { Se presenta una mayor } \\
\text { precisión sobre la pre- } \\
\text { dicción del conocimiento } \\
\text { de la estructura de la } \\
\text { máquina. }\end{array}$ & 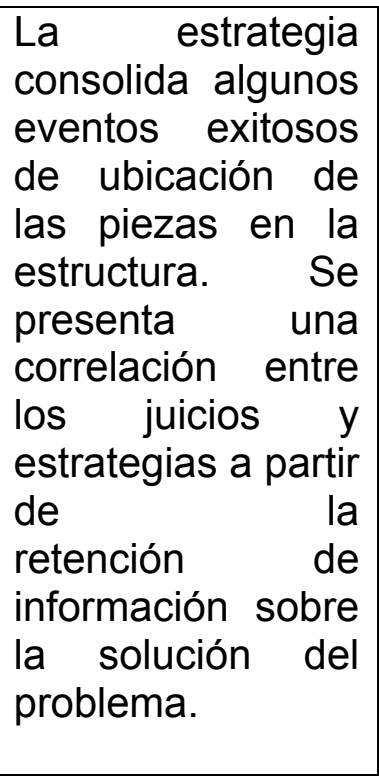 & $\begin{array}{lrr}\text { Afianza su nivel de } & \text { de } \\
\text { predicción sobre } & \text { el } \\
\text { manejo de la forma y } \\
\text { ubicación de las } \\
\text { piezas en } & \text { la } \\
\text { estructura. } & & \end{array}$ \\
\hline
\end{tabular}




\begin{tabular}{|c|c|c|c|}
\hline \multicolumn{4}{|l|}{ TERCER JUEGO } \\
\hline $\begin{array}{lr}\text { Acentúa } & \text { su } \\
\text { criterio } & \text { de } \\
\text { selección } & \text { para } \\
\text { seleccionar } & \text { la } \\
\text { segunda } & \\
\text { estrategia. } & \end{array}$ & $\begin{array}{l}\text { Recupere en un } 100 \% \text { la } \\
\text { información exitosa sobre } \\
\text { las piezas y su estructura. } \\
\text { La inversión para la } \\
\text { construcción de la } \\
\text { solución la reduce al } \\
\text { mínimo costo. }\end{array}$ & 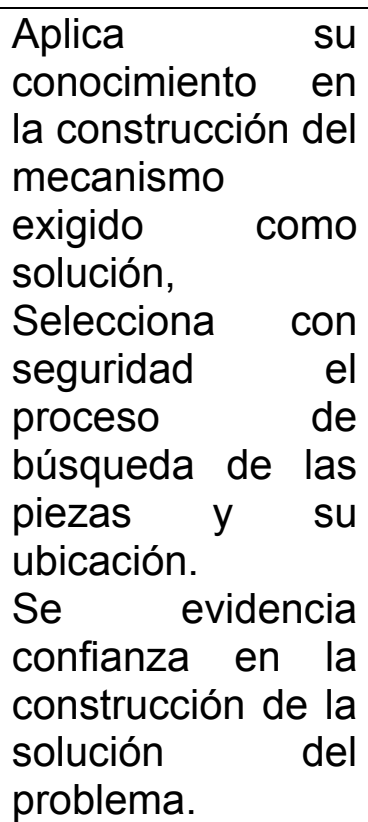 & $\begin{array}{l}\text { Reafirme su } \\
\text { conocimiento a partir } \\
\text { de la secuenciación } \\
\text { de los eventos } \\
\text { exitosos exigidos para } \\
\text { la construcción de la } \\
\text { Máquina. }\end{array}$ \\
\hline
\end{tabular}

Los grupos C y D en la etapa de generalización utilizan como punto de referencia constante la información textual o gráfica, tomándola como ejemplos de contrastación para la decisión que iban a tomar. Generan un plan de ensamblaje a partir de relacionar los escenarios de: selección de objetos; almacén y sitio de ensamble. Es evidente que para este grupo las producciones viejas se convierten en puntos de apoyo para lograr la construcción de producciones nuevas y resolver el problema. Con respecto al tiempo se debe resaltar que éste se incrementa considerablemente, pero, se mantiene constante el número de eventos para lograr cada éxito.

Las situaciones referidas anteriormente nos permiten esbozar algunas conclusiones sobre si existen diferencias significativas de los sujetos que lograron solucionar problemas a partir de optar por estrategias planteadas y los que no; de otra parte nos interesa explicar la diferenciación en términos del número de estrategias usadas y transferidas en las mismas situaciones descritas. Al respecto podemos afirmar las siguientes consideraciones:

1. Si entendemos los modelos como diseños y cuando son mentales los consideramos como elementos orientadores o dificultares de la comprensión del problema, podemos afirmar que: los sujetos que se enfrentaron únicamente con su propio modelo mental de máquina a resolver el problema presentaron una confrontación entre el modelo diseñado en el ambiente computacional y su propio modelo mental. Esta situación inhabilitó el avance en el proceso de comprensión, impidiéndolo un razonamiento basado en reglas de producción que lo llevara a la solución del problema.

2. La sugerencia de estrategia es un procedimiento diseñado para ayudar a realizar un determinado fin, sin contar con que garantice el éxito —un tipo de heurística- Se proyecta como una posibilidad de organización del pensamiento. Los grupos que contaron con la posibilidad de optar por una de las tres estrategias y de valorar su propio nivel de conocimiento hicieron uso consistente de la información textual y gráfica del ambiente de la tarea para mejorar la representación del problema. 
3. Con respecto a la transferencia de estrategias y a la utilización de éstas, se evidencia que los sujetos que siguieron consistentemente las sugerencias crearon con facilidad estrategias nuevas y fueren coherentes en el planeamiento de los procedimientos a utilizar para encontrar la solución, además logran mejorar los niveles de comprensión y utilización de todos los componentes del escenario experimental presentado.

\section{LA ORIENTACION}

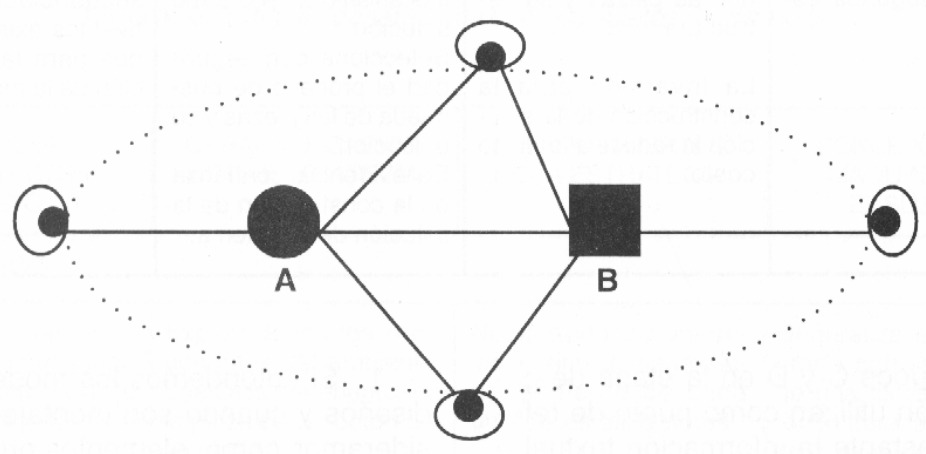

El Problema de orientación (Ilustración 2) consiste en hallar la posición desde la cual un observador tiene las imágenes correspondientes a dos objetos que aparecen simultáneamente. Los objetos A y B tienen posición fija en tanto el observador puede escoger su propia posición. El activador de juicios pide del jugador la previsión de tiempo para resolver el problema. Cuando el tiempo previsto por el jugador se agota, le exige una nueva previsión y reiniciar el juego. Hay tres estrategias disponibles para los grupos que cuentan con esta facilidad. El computador obliga elegir y leer una estrategia antes de iniciar.

Con base en los datos registrados y de acuerdo con el modelo de orientación definido por Hernández (1995), podemos realizar la siguiente caracterización de los dos grupos:

Con respecto al grupo que no cantó con sugerencias de estrategias se observa que los sujetos, en primera instancia, presentan un aumento progresivo de la eficacia vista a través de las etapas de aprendizaje. Entre la etapa de descubrimiento y las dos de consolidación se puede observar que los sujetos encontraron los eventos exitosos con una habilidad cada vez mayor a medida que se avanzaba en el juego. En la etapa de generalización se presenta el máximo nivel de eficacia.

En cuanto a eficiencia se presenta un aumento progresivo en las tres etapas. Se inicia con un manejo del tiempo calculado en los topes máximos y a medida que avanza en las etapas de aprendizaje va regulando su utilización y la de los clics para la búsqueda del evento exitoso. En la generalización esta relación muestra una ligera disminución comparada con la etapa de consolidación 2.

Con respecto a la construcción de estrategias de solución de problemas, el grupo no necesitó sugerencias sino que las construyó a partir del manejo de los componentes del problema, especialmente de los ejemplos objeto de simulación.

El grupo que jugó con sugerencias de estrategias no mantiene ninguna regularidad en cuanto a la relación éxitos-eventos, pero sí en cuanto a la relación éxitos-tiempo. Para la etapa de generalización se presenta una ligera disminución en los indicadores de eficacia y eficiencia. 
Los sujetos en las etapas del proceso de aprendizaje difícilmente relacionan los tres elementos del mareo de referencia del problema que son: objeto a ubicar, objeto de referencia y punto de vista. Se dedican a buscar relaciones entre la forma y el tamaño de los segmentos visibles sin tener en cuenta su orientación ni posición.

La relación entre tamaño y forma de los objetos incluidos en el problema para este grupo fue consolidándose como un elemento importante para la construcción de un modelo de solución. Este se desarrolló en la medida en que los sujetos comparaban el objeto ubicado con relación al objeto de referencia. Los sujetos de Los cuatro grupos, realizaron exploraciones de búsqueda del observador, manejando el espacio de trabajo, señalado como ventana 1 , desde diferentes formas:

- Una primera representación del proceso de búsqueda se realizó tomando uno de los segmentos como objeto primario y su respectivo ángulo visual como el objeto de referencia. La posición del clic es tomada como punto de vista del observador.

- La segunda representación se da con los mismos elementos de la anterior, pero difiere en que los sujetos tratan de realizar la integración de los componentes del problema tomando como objeto ubicado una de las composiciones determinadas por un segmento y su respectivo ángulo visual y, luego, observando si el objeto de referencia corresponde con la disposición del otro arreglo. El punto de vista sigue siendo el mismo de los casos anteriores. Para el problema planteado, el sujeto abstrae la forma y el tamaño de los indicadores objetivo del problema para definir su marco de referencia.

El análisis de transferencia de estrategias y del aprendizaje significativo para este problema, se realiza atendiendo a los siguientes componentes propios de la inteligencia espacial:

- Habilidad para reconocer instancias del mismo elemento.

- Habilidad para transformar o reconocer una transformación de un elemento en otro.

- Capacidad para evocar la imaginería mental y transformarla.

- Capacidad para producir una semejanza gráfica de información espacial.

Los contrastes de los datos referidos y organizados en las diferentes tablas se analizan teniendo en cuenta los planteamientos propios de la imaginería mental, entendida como la habilidad para resolver problemas derivados de la relación entre la imagen interna de los sujetos y la imagen vista en el mundo real. La estructura y el nivel de abstracción del problema exigían que los sujetos debieran recordar información acerca de las propiedades físicas de los objetos, vistas desde la posición y marco de orientación de los segmentos, información que se iba obteniendo en el momento, para ser codificada y aplicada a la construcción del modelo de solución en el marco de la orientación espacial.

Con base en el marco de referencia y el análisis de la información esbozamos las siguientes conclusiones:

1. Hay mayor promedio de eficacia sin estrategias que con estrategias. Los sujetos demostraron capacidad para relacionar en forma gráfica los componentes de las dos instancias del problema, situación que les permitió trabajar más al azar que consolidar una secuencia de eventos que los lleve a entender las relaciones de orientación en un espacio 
bidimensional y construir a través de las rotaciones mentales procedimientos secuenciales propios de un modelo de solución.

2. Hay mayor promedio de eficiencia con estrategias que sin estrategias. Debido a la dificultad para reconocer transformaciones de los objetos del problema y para identificar diferentes posiciones del mismo elemento -el observador- los sujetos debían entender el lenguaje de la estrategia sugerida e incorporarla como elemento constitutivo del problema. Esta situación que hace que se presente mayor eficacia, o sea mayor habilidad para encontrar el clic exitoso en el trabajo libre que en el trabajo desarrollado con el seguimiento de procedimientos sugeridos por la estrategia seleccionada.

3. Las estrategias jugaron un papel importante en el manejo del tiempo: se convirtieron en elementos reguladores de la relación eventos-tiempo. Si bien sirvieron para lograr un efecto positivo en a economía del tiempo, con relación a los eventos sucedió el efecto contrario, es decir, la cantidad de eventos se incrementó considerablemente.

\section{ANÁLISIS CUANTITATIVO DE RESULTADOS}

\section{Papel de los activadores de juicios de metamemoria y de las sugerencias de estrategias}

El análisis cuantitativo se hizo con base en datos tomados de ocho juegos, los cuales fueron organizados en un paquete que permitía acceso secuencial y controlado por el computador a través de un menú:

- Juego 1. Líneas en Equilibrio: hallar una línea oculta en ubicada en las zonas de equilibrio perceptivo, según los planteamientos de Arheim (1971). Los activadores estuvieron constituidos por una previsión de eventos requeridos y las estrategias fueron seleccionadas de una lista de tres con la exigencia de lectura de la misma antes de iniciar el juego y con disposición de la misma en todo momento a lo largo del juego.

- Juego 2. Líneas al Azar: buscar una línea oculta en cualquier zona de un espacio bidimensional. Los activadores de juicios de metamemoria y sugerencias de estrategias son comunes con los juegos 1, 3 y 4.

- Juego 3. Puntos en Equilibrio: Buscar un punto ubicado en las zonas de equilibrio perceptivo, según los planteamientos de Arheim (1971). Los activadores de juicios de metamemoria y sugerencias de estrategias son comunes con los juegas 1, 2 y 4.

- Juego 4. Puntos al Azar: hallar un punto en cualquier posición de un espacio bidimensional. Los activadores de juicios de metamemoria y sugerencias de estrategias son comunes con los juegos 1,2 y 3 .

- Juego 5. Rompecabezas de Piezas Ensamblables: armar la representación gráfica de la estructura inducida en un proceso de percepción Arheim (1971) usando un conjunto de piezas disponible, las cuales se pueden mover a una zona de ensamble. Los activadores solicitan un juicio sobre la facilidad de la tarea y una previsión de intentos requeridos para solucionar el problema. La sugerencia de estrategia fue seleccionada de una lista de tres con la exigencia de lectura de la misma antes de iniciar el juego y con disposición de la misma en todo momento a lo largo del juego. 
- Juego 6: Rompecabezas Vega JG. Consistió en armar la el cuadra usando el conocido rompecabezas de piezas deslizables. Este juego es común a todos los grupos y se toma como prueba de generalización frente a los juegos anteriores.

- Juego 8: Laboratorio de Color. El juego consistió en lograr una clase de efecto - sensación de volumen - mediante combinación de colores. Los activadores levan a hacer previsión de eventos y número de eventos requeridos para resolver el problema. Se cuenta con dos estrategias de las cuales se escoge una después de leerlas. El juego 84 es una variante de forma para el mismo juego 8 y se tomó para evaluar la generalización y es común en sus rasgos para los cuatro grupos.

Tabla 5. Resumen de diferencias significativas $-\mathrm{a}=0.05-$

\begin{tabular}{|c|c|c|c|c|c|c|c|c|c|c|c|c|c|c|c|c|c|c|c|c|c|c|}
\hline & & 11 & 12 & 13 & 21 & 22 & 23 & 31 & 32 & 33 & 41 & 42 & 43 & 51 & 52 & 53 & 61 & 81 & 82 & 83 & 84 & $\mathrm{~T}$ \\
\hline \multirow[t]{4}{*}{ Eficacia } & C & - & + & + & - & - & + & - & - & - & - & - & - & - & - & - & - & - & - & - & - & 3 \\
\hline & $A$ & - & + & + & - & - & - & - & - & - & + & - & - & - & + & - & - & - & - & - & + & 5 \\
\hline & $E$ & - & - & - & - & - & - & + & - & - & - & - & - & - & - & - & - & - & - & - & - & 1 \\
\hline & 2 & - & - & - & - & - & + & - & - & - & + & - & - & - & + & - & - & - & - & - & + & 4 \\
\hline \multirow[t]{4}{*}{ Eficiencia } & $\mathrm{C}$ & - & + & - & - & - & + & + & - & - & + & + & + & + & - & - & - & - & - & - & - & 7 \\
\hline & $\mathrm{A}$ & - & + & - & - & - & - & - & - & - & - & - & - & & - & - & - & - & - & - & - & 1 \\
\hline & $E$ & - & - & - & - & - & - & - & - & + & - & - & + & + & + & - & - & - & - & - & - & 4 \\
\hline & 2 & - & - & - & - & - & + & - & - & - & + & - & - & - & - & - & - & - & + & - & + & 4 \\
\hline
\end{tabular}

Se tomaron datos en tres momentos del mismo juego que denominamos descubrimiento —el sujeto resuelve el juego por primera vez-, consolidación 1segunda solución del juego -y consolidación 2- última sesión con cada juego. En las tablas 5 a 9 el primer dígito que encabeza cada juego es el número de juego y el segundo dígito corresponde a la etapa (descubrimiento $=1$, consolidación $1=2$ y consolidación $2=$ 3).

Los resultados fueron comparados usando Análisis Factorial de Varianza para cada uno de los juegos y etapas del aprendizaje, con un nivel de significación de 0.05.

La tabla 5 muestra los juegos en los cuales el análisis de varianza muestra diferencias significativas entre los grupos - señalado con el signo +- y los grupos que son significativamente superiores. La última columna representa un canteo del número de veces en que el grupo se mostró significativamente superior a los otros. Se pueden contrastar las significaciones tanto para la variable dependiente eficacia como eficiencia.

La tabla 6 desagrega los resultados para la etapa de descubrimiento.

Tabla 6. Significación en etapa de descubrimiento.

\begin{tabular}{|l|l|l|l|l|l|l|l|l|l|}
\hline & & 11 & 21 & 31 & 41 & 51 & 61 & 81 & $T$ \\
\hline Eficacia & Control & - & - & - & - & - & - & - & 0 \\
\cline { 2 - 9 } & Activadores & - & - & - & + & - & - & - & 1 \\
\cline { 2 - 9 } & S.Estrategias & - & - & + & - & - & - & - & 1 \\
\cline { 2 - 9 } & Activadores \& S.Estategias & - & - & - & + & - & - & - & 1 \\
\hline \multirow{5}{*}{ Eficiencia } & Control & - & - & + & + & + & - & - & 3 \\
\cline { 2 - 9 } & Activadores & - & - & - & - & & - & - & 0 \\
\cline { 2 - 9 } & S.Estrategias & - & - & - & - & + & - & - & 1 \\
\cline { 2 - 8 } & Activadores \& S.Estategias & - & - & - & + & - & - & - & 1 \\
\hline
\end{tabular}


La tabla 7 desagrega los resultados para la etapa de consolidación 1.

Tabla 7. Significación en etapa de consolidación 1.

\begin{tabular}{|l|l|l|l|l|l|l|l|l|}
\hline & & 12 & 22 & 32 & 42 & 52 & 82 & $T$ \\
\hline \multirow{4}{*}{ Eficacia } & Control & + & - & - & - & - & - & 1 \\
\cline { 2 - 8 } & Activadores & + & - & - & - & + & - & 2 \\
\cline { 2 - 8 } & S.Estrategias & - & - & - & - & - & - & 0 \\
\cline { 2 - 8 } & Activadores \& S.Estategias & - & - & - & - & + & - & 1 \\
\hline \multirow{5}{*}{ Eficiencia } & Control & + & - & - & + & - & - & 2 \\
\cline { 2 - 8 } & Activadores & + & - & - & - & - & - & 1 \\
\cline { 2 - 8 } & S.Estrategias & - & - & - & - & + & - & 1 \\
\cline { 2 - 8 } & Activadores \& S.Estategias & - & - & - & - & - & + & 1 \\
\hline
\end{tabular}

La tabla 8 desagrega los resultados para la etapa de consolidación 1.

Tabla 8. Significación en la segunda etapa de consolidación.

\begin{tabular}{|l|l|l|l|l|l|l|l|l|}
\hline & & 13 & 23 & 33 & 43 & 53 & 83 & $T$ \\
\hline \multirow{5}{*}{ Eficacia } & Control & + & + & - & - & - & - & 2 \\
\cline { 2 - 8 } & Activadores & + & - & - & - & - & - & 1 \\
\cline { 2 - 8 } & S.Estrategias & - & - & - & - & - & - & 0 \\
\cline { 2 - 8 } & Activadores \& S.Estategias & - & + & - & - & - & - & 1 \\
\hline \multirow{5}{*}{ Eficiencia } & Control & - & + & - & + & - & - & 2 \\
\cline { 2 - 8 } & Activadores & - & - & - & - & - & - & 0 \\
\cline { 2 - 8 } & S.Estrategias & - & - & + & + & - & - & 2 \\
\cline { 2 - 8 } & Activadores \& S.Estategias & - & + & - & - & - & - & 1 \\
\hline
\end{tabular}

En la tabla 9 se coloca el número de orden obtenido al comparar las medias de cada uno de los grupos en cada juego, según cada una de las variables de pendientes. La penúltima columna calcula la suma de estos puntajes de ordenamiento y la última columna considera la media de las mismas puntuaciones.

Tabla 9. Asignación de puntos por grupo según media estadística en todos los juegos.

\begin{tabular}{|c|c|c|c|c|c|c|c|c|c|c|c|c|c|c|c|c|c|c|c|c|c|c|c|}
\hline & & 11 & 12 & 13 & 21 & 22 & 23 & 31 & 32 & 33 & 41 & 42 & 43 & 51 & 52 & 53 & 61 & 81 & 82 & 83 & 84 & $\mathrm{St}$ & $\mathrm{Mt}$ \\
\hline \multirow[t]{4}{*}{ Eficacia } & Cont & 2 & 2 & 3 & 1 & 3 & 1 & 3 & 2 & 2 & 2 & 2 & 3 & 1 & 1 & 1 & 1 & 4 & 1 & 3 & 2 & 40 & 2 \\
\hline & Act & 4 & 4 & 4 & 1 & 4 & 3 & 1 & 3 & 1 & 3 & 3 & 1 & 2 & 2 & 3 & 4 & 1 & 2 & 4 & 3 & 54 & 3 \\
\hline & Est & 1 & 1 & 0 & 4 & 2 & 2 & 4 & 4 & 4 & 1 & 1 & 1 & 3 & 3 & 2 & 2 & 2 & 3 & 1 & 1 & 42 & 2 \\
\hline & A\&E & 3 & 3 & 2 & 3 & 1 & 4 & 3 & 1 & 3 & 4 & 4 & 4 & 4 & 4 & 4 & 3 & 3 & 4 & 2 & 4 & 63 & 3 \\
\hline \multirow[t]{4}{*}{ Eficiencia } & Cont & 2 & 3 & 4 & 1 & 4 & 1 & 4 & 4 & 3 & 4 & 4 & 4 & 3 & 3 & 4 & 3 & 4 & 2 & 1 & 1 & 59 & 3 \\
\hline & Act & 4 & 4 & 3 & 1 & 2 & 2 & 2 & 1 & 1 & 3 & 3 & 3 & 1 & 1 & 1 & 1 & 1 & 1 & 2 & 2 & 39 & 2 \\
\hline & Est & 2 & 1 & 1 & 4 & 2 & 2 & 3 & 3 & 4 & 1 & 1 & 1 & 4 & 4 & 3 & 2 & 1 & 2 & 2 & 2 & 45 & 2 \\
\hline & A\&E & 1 & 2 & 2 & 1 & 1 & 4 & 1 & 2 & 2 & 2 & 1 & 1 & 2 & 3 & 2 & 3 & 1 & 4 & 4 & 4 & 43 & 2 \\
\hline
\end{tabular}

La tabla 10 se desagrega la tabla 9 para una mejor comparación de los juegos por etapas y las etapas entre si. 
Tabla 10. Media de puntos asignados por grupo según media estadística por etapas.

\begin{tabular}{|l|l|l|l|l|l|l|l|l|l|}
\hline & & $\begin{array}{l}\text { Suma } \\
\text { total }\end{array}$ & $\begin{array}{l}\text { Media } \\
\text { total }\end{array}$ & $\begin{array}{l}\text { Suma } \\
\text { descub }\end{array}$ & $\begin{array}{l}\text { Media } \\
\text { descub }\end{array}$ & $\begin{array}{l}\text { Suma } \\
\text { cons. 1 }\end{array}$ & $\begin{array}{l}\text { Media } \\
\text { cons. 1 }\end{array}$ & $\begin{array}{l}\text { Suma } \\
\text { cons. 1 } 1\end{array}$ & $\begin{array}{l}\text { Media } \\
\text { cons. } 1\end{array}$ \\
\hline Eficacia & Control & 40 & 2 & 13 & 2 & 11 & 2 & 13 & 2 \\
\cline { 2 - 10 } & Activadores & 54 & 3 & 12 & 2 & 19 & 3 & 16 & 3 \\
\cline { 2 - 10 } & S.Estrategias & 42 & 2 & 15 & 3 & 13 & 2 & 11 & 2 \\
\cline { 2 - 10 } & $\begin{array}{l}\text { Activadores \& } \\
\text { S.Estategias }\end{array}$ & 63 & 3 & 20 & 3 & 17 & 3 & 19 & 3 \\
\hline Eficiencia & Control & 59 & 3 & 18 & 3 & 20 & 3 & 17 & 3 \\
\cline { 2 - 10 } & Activadores & 39 & 2 & 12 & 2 & 12 & 2 & 12 & 2 \\
\cline { 2 - 10 } & S.Estrategias & 45 & 2 & 15 & 3 & 13 & 2 & 13 & 2 \\
\cline { 2 - 10 } & $\begin{array}{l}\text { Activadores \& } \\
\text { S.Estategias }\end{array}$ & 43 & 2 & 8 & 1 & 13 & 2 & 15 & 3 \\
\hline
\end{tabular}

La ilustración 3 compara de manera global los resultados de las cuatro condiciones experimentales.

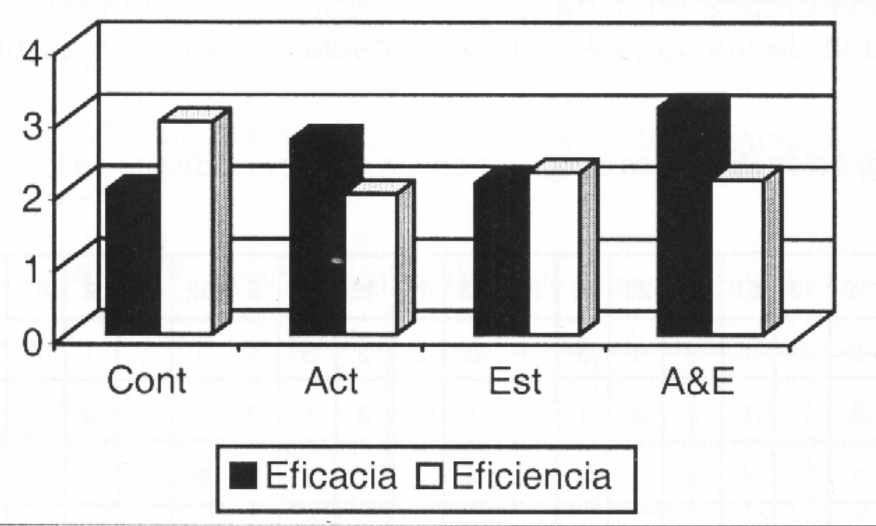

Ilustración 3. Comparación de los grupos e Eficiencia y Eficiencia.

\section{ESTRATEGIAS FUERTES Y TRANSFERENCIA DE ESTRATEGIAS}

La tabla 11 muestra las relaciones significativas entre predictor y predicción discriminadas para las dos variables dependientes como factores explicados. Se utilizan como predictores los valores en las tres etapas del juego predictor y la variable grupos - control, con activadores de juicios de metamemoria, con sugerencia de estrategias y con activadores y sugerencia combinados-; la variable dependiente se mide en la etapa de descubrimiento del juego de la columna rotulada "Predicción".

En el análisis de la eficacia como factor explicado se encuentra que se da una relación significativa entre los puntajes en eL tercer intento de la solución de los juegas Agujeros en Equilibrio y el primero de Agujeros al Azar y de manera similar entre el segundo y tercer intentos de el primer juego de Color y el primer intento en el segundo juego de Color. Las otras relaciones no son significativas.

Las relaciones en la columna eficiencia son más abundantes. La variable grupo se muestra como predictor significativo en tres de los cinco casos analizados, e igualmente los resultados en etapa de descubrimiento son predictores significativos en tres de Los cinco casos. Los resultados en segundo y tercer intento de solución de los juegos no son predictores significativos para eficiencia. 
Tabla 11. Relaciones significativas en el análisis de regresión.

\begin{tabular}{|l|l|l|l|}
\hline Predictor & Predicción & Eficacia & Eficiencia \\
\hline Líneas en equilibrio & Líneas al azar & No significativa & Descubrimiento \\
\hline Líneas en equilibrio & $\begin{array}{l}\text { Agujeros en } \\
\text { equilibrio }\end{array}$ & No significativa & Grupos \\
\hline $\begin{array}{l}\text { Agujeros en } \\
\text { equilibrio }\end{array}$ & Agujeros al azar & Consolidación 2 & $\begin{array}{l}\text { Descubrimiento y } \\
\text { grupos }\end{array}$ \\
\hline $\begin{array}{l}\text { Rompecabezas } \\
\text { Arheim }\end{array}$ & $\begin{array}{l}\text { Rompecabezas } \\
\text { Vasarely }\end{array}$ & No significativa & Descubrimiento \\
\hline Color Juego 1 & Color Juego 2 & $\begin{array}{l}\text { Consolidación 1 y } \\
\text { Consolidación 2 }\end{array}$ & Grupos \\
\hline
\end{tabular}

\section{CONCLUSIONES}

\section{Papel de los activadores de juicios de metamemoria y de las sugerencias de estrategias}

Tanto los activadores de juicios de metamemoria como las sugerencias de estrategias tienden a ser más efectivos después de alguna experiencia de base en la solución de los problemas, resultados que son coherentes con los hallazgos de investigación previa que muestran que los estudiantes novicios en una materia tienen menor nivel de metacognición. Se puede afirmar que el papel de las dos variables se incrementa con el avance en la curva del aprendizaje, pero, también que otras condiciones de entrenamiento - caso del grupo control- pueden tener efectos similares en etapas avanzadas del entrenamiento. La combinación de activadores de juicios y estrategias genera un efecto de interacción positivo que hace que a través de las diferentes etapas el grupo que combina los dos factores tienda a tener puntajes entre intermedio y alto.

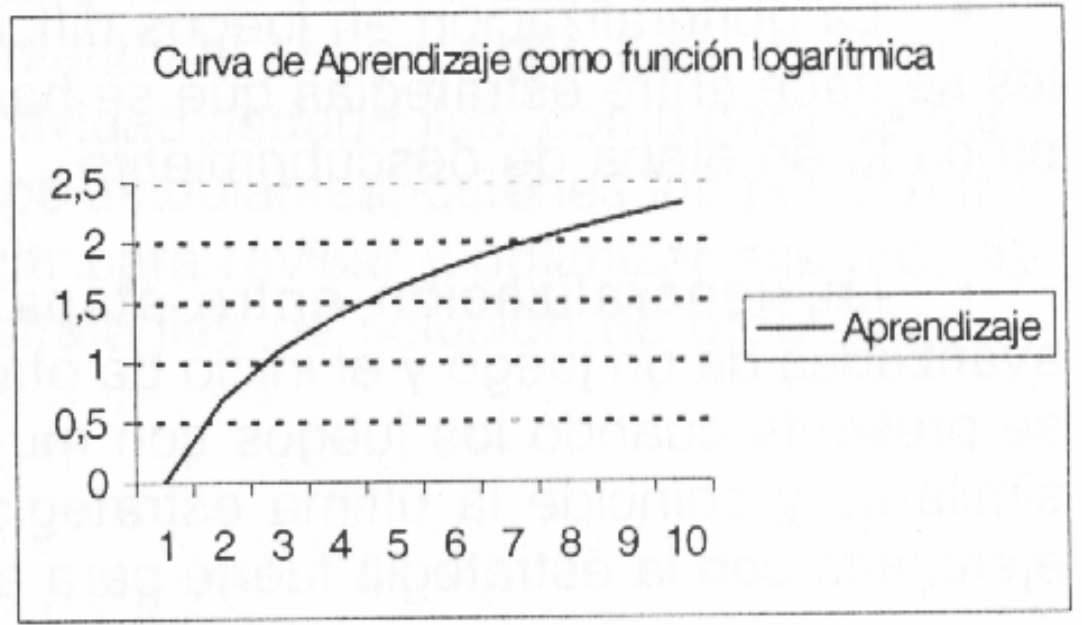




\section{Relación entre estrategias fuertes y generalización}

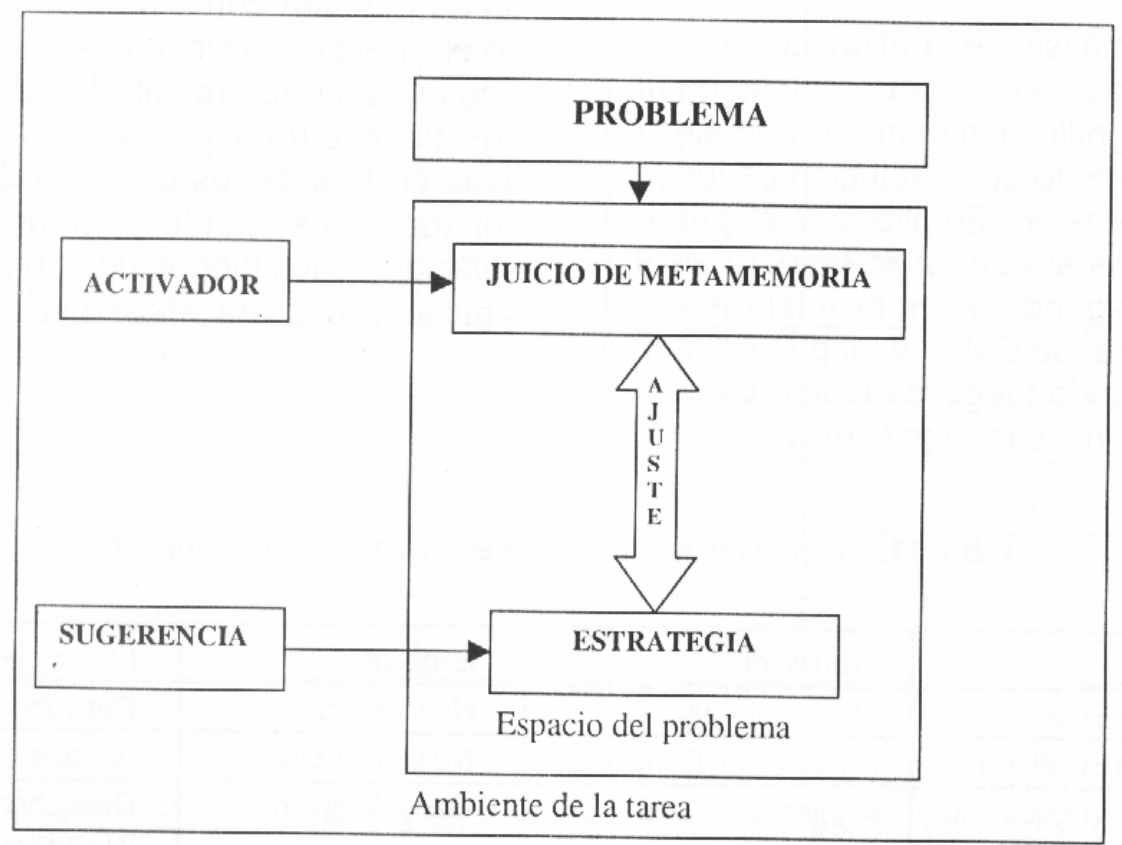

Gráfica 1. Papel de los activadores de juicios de metamemoria y las sugerencias de estrategias en la solución de problemas

Los resultados sobre estrategias fuertes y su generalización encontrados en este estudio se pueden resumir en las siguientes aseveraciones:

- La diferencia en generalización cuando se toma la eficacia y la eficiencia...

- Los grupos que combinan juicios y estrategias tienden a desarrollar mayores niveles de generalización.

- La generalización en juegos difíciles se hace entre estrategias que se han probado en etapa de descubrimiento.

- La generalización entre etapas avanzadas de un juego y el inicio de otro se presenta cuando los juegos son muy similares y coincide la última estrategia ejercitada con la estrategia fuerte para el siguiente juego; en otros casos la correlación de este factor es negativa mostrando dificultad para la generalización.

- Cuando se desarrollan niveles altos de rendimiento en un juego y se enfrenta uno nuevo que no es posible de resolver con la estrategia prevaleciente en el primero, el nuevo aprendizaje se torna más difícil.

El hallazgo de resultados de transferencia alta cuando se centra la solución del primer problema en una estrategia válida para el segundo, se puede explicar porque la última estrategia probada como muy exitosa en el juego anterior se convierte en la primera utilizada para enfrentar el nuevo juego. Si la estructura del juego hace que esta estrategia sea nuevamente exitosa, la transferencia es alta y positiva; de lo contrario gastará esfuerzo proporcional a su seguridad en refutarla, lo cual se muestra en el valor negativo de la correlación. 
La afirmación en pedagogía de que se debe enseñar partiendo de lo simple para llegar a lo complejo, eventualmente puede ser productiva si la estrategia exitosa en el último problema corresponde a una estrategia exitosa en el siguiente; pero, no necesariamente esta forma de proceder es la más fácil para el aprendiz.

\section{IMPORTANCIA DE LOS JUEGOS DE DESCUBRIMIENTO EN EL APRENDIZAJE AUTÓNOMO}

Ausubel, Novack y Hanesian (1973) distinguen en el aprendizaje significativo el aprendizaje por descubrimiento dirigido y el autónomo. Los ambientes de descubrimiento son fundamentales en la formación de la autonomía del aprendizaje en contraposición a los ambientes ricos en orientaciones y explicaciones, por cuanto debe someter sistemáticamente a prueba lo que tiene en memoria de largo plazo y construir o incrementar nuevos conceptos y estrategias que enriquezcan su conocimiento previo, mediante una serie de decisiones que están solamente bajo su control. Las mismas sugerencias que pueden recibir en el ambiente de descubrimiento tienen condiciones de contrastación que disminuyen el potencial de autoridad inherente a las comunicaciones codificadas orales o escritas. En la medida que se incrementan las instrucciones y se disminuye la actividad de búsqueda o de exploración del entorno, los sujetos dedican más tiempo a interpretar y seguir instrucciones que a conocer el ambiente y valorar su propia actividad de búsqueda.

\section{LOS SIMULADORES Y ESTUDIO DE PROCESOS EN EL APRENDIZAJE}

La versión digital de protocolos — simuladores- que se desarrolló permite, además de la replica de procesos, aplicar técnicas computacionales para exploración de datos y tomar secuencias que pueden ser analizadas de diferentes maneras. La identificación, seguimiento y orientación de los procesos de aprendizaje es una meta propuesta pero no alcanzada en la Pedagogía Contemporánea. Los simuladores contribuyen al logro de este propósito, compitiendo con alternativas analógicas como es el caso del vídeo o la grabación de audio.

\section{Proyecciones y limitaciones}

Aparentemente las sugerencias de estrategias serían más productivas cuando el sujeto ya ha probado las estrategias que tiene almacenadas en memoria de largo plazo, por lo cual sería de importancia comparar la efectividad de estrategias sugeridas al iniciar la solución del problema con estrategias que se activan cuando se pueda inferir que ha validado estrategias no productivas que trae de su experiencia anterior.

Especial interés merece la utilización de simuladores para la replicación, descripción, análisis y optimización de procesos de solución de problemas. Su proyección se perfila tanto para el entrenamiento de profesores, los cuales pueden utilizar estos dispositivos para aprender sobre los procesos seguidos por los estudiantes y por expertos y así orientar su actividad pedagógica, como para los mismos estudiantes, quienes los pueden utilizar para revisar y optimizar sus propias estrategias de solución de problemas. 


\section{REFERENCIAS BIBLIOGRÁFICAS}

.Arheim R. 97). Arte y percepción visual. Madrid: Alianza Ed., primera edición, 1971.

Ausubel, D.P., Novack, J. Y Hanesian H. (1973). Educational Psychology. New York: Holt, Rinehart \& Wiston.

Fayyad, U.M. et al. (1996). Advances in Know!edge Discovery and Data Mining. Cambridge, MA: AAAI Press / The MIT Press.

Hernández, D. (1995). Qualitative representation of spatial knocoledge. New York: Springer vertag.

Maldonado, G.,L.F. y Andrade L., Édgar (1996). Ambiente computarizado para el aprendizaje autodirigido del diseño -ACA2- Bogotá: Universidad Pedagógica Nacional, Proyecto de Investigación convenio Colciencias UPN.

Nelson T. O., \& Narens, L. (1990). Metamemory: A Theoretical Framework and new Fidings. In METCALFE,J. \& SHIMAMURA, A. P.(Eds). Metacognition. Cambridge, MA: The MIT Press. Preface.

Stenberg, R.J. (1984). Toward Triarchic Theory of Human Intelligencie. The brain an Behavioral Sciences, 7, 2-69-315. 